\title{
The Role of Pig Production and Market Value Chain in the Occurrence of African Swine Fever in Songwe and Ruvuma Regions, Tanzania
}

\author{
Ntuli Ngosomwilea, Calvin Sindato ${ }^{a, b}$, Erick V.G. Kombac, Daniel W. Ndyetabula ${ }^{d}$ \\ aSACIDS Foundation for One Health, Africa Centre of Excellence for Infectious Diseases of Human and Animals, Sokoine University of Agriculture, P.O. Box 3297, \\ Chuo Kikuu, Morogoro, Tanzania. \\ bNational Institute for Medical Research, Tabora Research Centre, P.O. Box 482, Tabora, Tanzania. \\ Department of Veterinary Medicine and Public Health, College of Veterinary Medicine and Biomedical Sciences, Sokoine University of Agriculture, P.O. Box \\ 3021, Chuo Kikuu, Morogoro, Tanzania. \\ dDepartment of Agricultural Economics and Agribusiness, School of Agribusiness, Sokoine University of Agriculture, P. O. Box 3007, Chuo Kikuu, Morogoro, \\ Tanzania. \\ * Correspondence to ntuli.mwakilembe@sacids.org (Ntuli Ngosomwile)
}

\section{ABSTRACT}

Background: In recent years, the pig industry in Tanzania has faced frequent occurrence of outbreaks of African swine fever (ASF). However, there is inadequate information on the pig value chain operation in relation to occurrence of ASF. This study aimed at mapping pig value chain and assess its contribution to the occurrence and spread of ASF in Tanzania.

Methods: A cross sectional study was carried out in Songwe, Momba, Songea and Mbinga districts of Tanzania. Study districts were purposively selected based on the density of pig population, differences in production systems and history of ASF outbreaks. A total number of 484 pig producers and 28 traders were involved in the study. Random sampling was used to select pig producers. Pig traders were selected using snowball technique. Structured questionnaires were used to collect data on pig management and production practices, veterinary services, pig marketing practices and biosecurity measures using Open Data Kit (ODK) software. Semi structured interviews were conducted with key informants on perceived risk practices that are related to ASF outbreaks, challenges and recommendations on ASF prevention and control measures. Observation method was used to assess structure, facilities and practices within the pig production chain.

Results: The main actors in the pig production chain were pig producers, assemblers, wholesalers and retailers. Unknown stock source $(30 \%)$, poor husbandry practice such as free ranging $(5 \%)$, poor management of waste products $(73 \%)$ and poor handling of feed $(73 \%)$ were risk practices in the production node. Transportation nodes operated under high risk due to frequent movements and pick-ups of $\geq 30$ pigs per trip.

Conclusion: The results demonstrated that different actors operate in the pig production, distribution and marketing chain. Each node operated under low biosecurity measures, and poor infrastructures that are likely to contribute to occurrence of ASF. There is need to improve good husbandry practices, marketing and infrastructures to increase production while ensuring pork safety.

Keywords: African swine fever; animal protein; pig value chain; Tanzania

\section{INTRODUCTION}

Jlobally, pig production is increasingly driven by

the demand for animal protein in the market. The pig industry accounts for 118.7 million tonnes of pork meat of which a large amount is produced in China, European Union countries, the United States of America, Brazil and Russia. ${ }^{1}$ The world pork meat market has increased by $1.4 \%$ from 8.1 million tonnes in 2017 to 8.3 million tonnes in $2018 .^{2}$ In Sub-Saharan Africa (SSA), South Africa accounts for $26 \%$ of the pig 
production followed by Nigeria (19\%) and Uganda $(12 \%) .^{3}$ Available statistics indicate that there are approximately 2 million pigs in Tanzania. ${ }^{4}$ The consumption of pork meat is increasing due to the price of substitute meat products, increasing wealth, and growing population in both rural and urban centers. ${ }^{5}$ Parallel to this, Tanzania's pig industry has the capacity to grow and reduce a productionconsumption deficit for pork from 8000 to 1350 tonnes in 2025. ${ }^{4}$ Despite this rapid growth of the sector its economic contribution is threated by outbreaks of African swine fever (ASF). ${ }^{6}$

ASF is an infectious disease and number one killer of pig populations. ${ }^{4}$ The disease is caused by Deoxyribonucleic acid (DNA) virus that belongs to the genus Asfivirus of the family Asfarviridae. ${ }^{7}$ It is a major constraint in development of pig industry in Europe, Asia and Africa due to a mortality rate of up to $100 \%$ in domestic pigs. ${ }^{8}$ In sub Saharan Africa, over 22 countries have reported ASF epidemics. ${ }^{6}$ The disease was first reported in Eastern African countries in $1921 .{ }^{9}$ In the absence of a vaccine, ASF control has relied on culling/ slaughtering of pigs, proper disposal of carcasses, quarantine, sanitation and hygiene measures and education interventions. ${ }^{10}$

The prevention and control of ASF depends on available information on chains operation and performance using value chain analysis. ${ }^{11}$ The analysis describes the system dynamic, classifying interaction and linkages as well as assess the behavioral risk practices such feeding swill, farm visitors, sharing boars and free ranging which contribute to disease transmission. ${ }^{11}$ Understanding of the pig value chain, drivers and factors contributing to transboundary animal disease spread can contribute to designing of effective control strategies. Value chain refers to a full range of activities which are required to bring products or services from production, marketing channels to end consumers and final disposal after use. ${ }^{12}$ It can be classified based on two approaches: The first approach is based on the role of chain governance and the second approach on coordination and operation of activities within chains. The former determines the rule of trade and who decides what to produce (producers' or buyers' chain) while the latter defines how people or actors in value chain perform and the decisions they make. ${ }^{13,14}$

While previous studies in Tanzania have reported on ASF epidemiology and molecular identification of African swine fever virus (ASFV) genotypes circulating in different parts of the country, ${ }^{15,16,17}$ there is dearth of information on the role played by pig value chain operation in disease epidemiology. Such information could contribute in designing effective ASF disease management within the country. Therefore, the objective of this study was to map pig value chain and assess its role in the occurrence and spread of ASF in selected districts of Tanzania.

\section{METHODS}

\section{Study Area}

The study was conducted in Songwe and Ruvuma regions of southern Tanzania. In Songwe two districts, Momba and Songwe were involved; while in Ruvuma, Mbinga district and Songea district were involved. The populations of Songwe, Momba, Songea and Mbinga districts are 133,692,196,818,173,821 and 353, 683 respectively. ${ }^{18}$ Crop and livestock production are the most important economic activities in these study areas. Additionally, the areas were purposively chosen based on different ecosystems of animal production, history of ASF outbreaks and high density of pig population; whereby Songwe region has approximately 79,513 pigs, ${ }^{19}$ and Ruvuma region has a population of 183,276 pigs. ${ }^{18}$ There are five veterinary health services (consultation and drug outlets) within study areas. In Songwe district selected wards and villages were Ifwenkenya ward (Ifwenkenya and Iyela villages) and Namkukwe ward (Namkukwe and Mheza villages); Momba district, Ikana ward (Ikana and Nyenjele villages) and Myunga ward (Namshinde and Mfuto villages); Songea district, Peramiho ward (Peramiho A village), Ngogosi ward (Namatuhi village), Kizuka ward (Ngahokora village), and Magagura ward (Magagura village); Mbinga district, Mbinga Mjini B ward (Misheni street), Luhuwiko ward (Luhuwiko A and Luhuwiko B streets), Bethelehemu ward (Mahela and Bethelehemu streets) and Lusonga ward (Ruvuma and Kihaha streets) (Fig 1). 


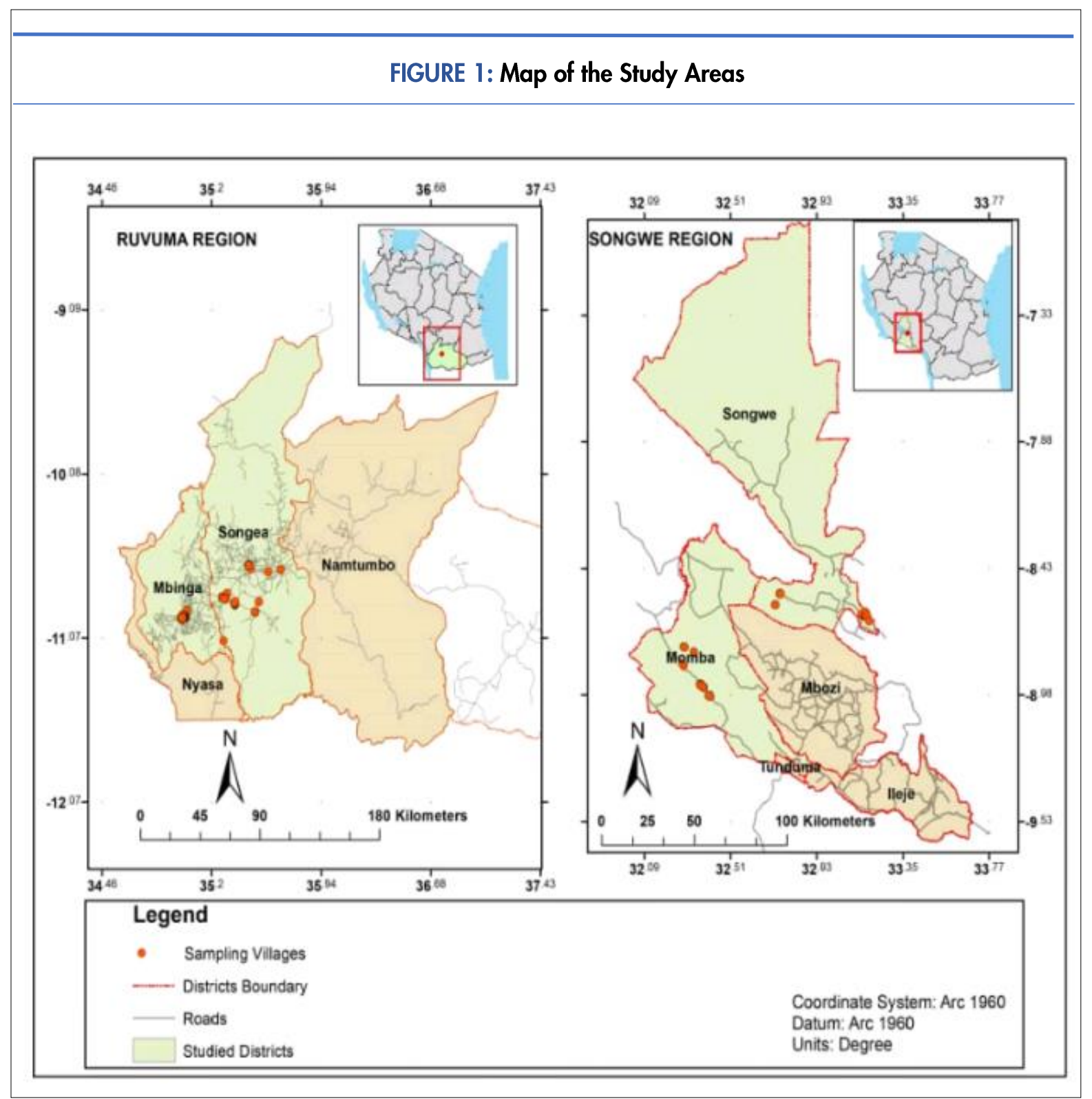

\section{Study Design and Sample Collection}

A cross sectional study was carried out in which data were collected from October to November 2018 in Songwe and Momba districts, and from June to July 2019 in Songea and Mbinga districts. The sample size for pig producers was determined by the formula developed by Kothari, ${ }^{20}$ i.e. $\mathrm{n}=\mathrm{z} 2 \mathrm{pq} / \mathrm{d}^{2}$ where by $\mathrm{n}$ is the sample size, $\mathrm{z}$ is 1.96 corresponding to the $95 \%$ confidence interval level and significant level of $5 \%$, p is the prevalence of disease which is estimated at $50 \%$ ${ }^{21}, \mathrm{q}$ is $1-\mathrm{p}$, and $\mathrm{d}$ is the permissible error of estimation (0.05). Therefore $1.96^{2} \times 0.5 \times 0.5 / 0.05^{2}=384$. A contingency of $26 \%$ was added to account for nonresponses and incomplete data was added resulting to a final sample size of 484 households keeping pigs. Since there were no physical markets for pigs in study districts, sampling of traders was based on known information, accessibility and availability of them during data collection. Thus, a total number of 28 pig traders were obtained from all surveyed districts.

Prior information on ASF outbreaks distribution in the study areas was obtained from respective district veterinary offices. Livestock Field officers provided lists of pig producers within study areas that served as sampling frames for pig producers. In this study we 
interviewed pig producers with and without pigs at the time of data collection. Snowball sampling technique was used to obtain traders within study areas. The information about the first trader came from field officers in villages, thereafter, the same trader was used to identify other fellow traders one after another for interviews and the process was repeated until the point of thematic saturation was reached. The targeted pig trader populations were assemblers, wholesalers and retailers.

The value chain in this study was based on coordination and operation of activities within the pig production system. An assumption was made that operations and activities in pig value chain are independent and absence of command within chains. ${ }^{22}$ In addition, this approach could enable assessment of risk practices in relation to transmission of disease within chains.

\section{Data Collection}

A semi-structured questionnaire was used to collect data from pig producers and pig traders. The questionnaire for pig producers focused on household characteristics, source of stocks, production system, production constraints, breeding type, herd size, feed materials, premises structures, management of waste products, training and veterinary services, and biosecurity measures. While the questionnaire for pig traders included questions on characteristics of pig traders, type of business, initial capital, source of stock, marketing prices, marketing distance, marketing constraints, management of waste products and biosecurity measures. Three research assistants were recruited and trained on study objectives and protocol. Software was used to collect data. The English version of a semi-structured questionnaire was translated to Kiswahili. The questionnaire was installed in the android mobile device with Open Data Kit software. It was pre-tested in an area in Songwe district that was not involved in the actual study. Modifications were made to the questionnaire before been used for actual data collection. A single interview took approximately 30 minutes to complete.

Moreover, we interviewed district livestock officers $(n=2)$ and field officers $(n=8)$ as key informants.
Furthermore, we directly observed the presence of veterinary services, water supply, means of animal transport, slaughter facilities, animal inspection, pig premises, presence of fence, ticks, warthogs, disposal of waste products and behavioral risk practices such as feeding swills, free ranging, farm visitors and sharing boars and farm equipment without disinfectant that could contribute to introduction and spread of ASF. These parameters were marked good practices as 1 , poor practices as 2 and not applicable as 3 .

\section{Statistical Data Analysis}

Data analysis was done using Statistical Package for Social Sciences (IBM SPSS) version $25^{\circledR}$ (IBM Corp, Armonk New York, USA). Data were summarized using descriptive statistics including percentage, median and frequency tables to describe general characteristic and graphical representation of key actors in pig value chain. For qualitative data thematic analysis was used whereby specific themes were deduced from qualitative research questions. Thematic areas addressed in this study include knowledge on risk practices that related to ASF outbreaks, opinion on factors limiting the control of ASF and recommendations on ASF prevention and control measures.

\section{Ethical Approval}

The study was approved by the Sokoine University of Agriculture (reference number SUA/CVMBS//R.1/2018/2019/2). Permission to conduct the study was obtained from the respective district and village authorities. Informed consent was obtained from pig owners.

\section{RESULTS}

A total of 484 respondents were involved in the study. Of these, 99 were from Songwe, 97 from Momba, 119 from Songea and 169 were from Mbinga. Of the total respondents, $45 \%$ were females and $55 \%$ males between minimum age 13 to maximum 85 years. Over $50 \%$ of pig producers had attained primary education as shown in Table 1. 
TABLE 1: Socio-demographic characteristics of pig producers in Songwe and Ruvuma regions

\begin{tabular}{|c|c|c|c|c|c|c|c|}
\hline \multirow[t]{2}{*}{ Category } & \multirow[t]{2}{*}{ Variables } & \multicolumn{3}{|c|}{ Songwe Region } & \multicolumn{3}{|c|}{ Ruvuma Region } \\
\hline & & $\begin{array}{l}\text { Songwe } \\
(n=99)\end{array}$ & $\begin{array}{l}\text { Momba } \\
(n=97)\end{array}$ & $\begin{array}{l}\text { Total } \\
(N=196)\end{array}$ & $\begin{array}{l}\text { Songea } \\
(n=119)\end{array}$ & $\begin{array}{l}\text { Mbinga } \\
(\mathrm{n}=169)\end{array}$ & $\begin{array}{l}\text { Total } \\
(\mathrm{N}=288)\end{array}$ \\
\hline & & No. (\%) & No. (\%) & No. (\%) & No. (\%) & No. (\%) & No. (\%) \\
\hline \multirow[t]{2}{*}{ Sex } & Male & $58(58.6)$ & $88(90.7)$ & $\begin{array}{c}146(74 \\
.5)\end{array}$ & $62(52.1)$ & $58(34.3)$ & $120(41.7)$ \\
\hline & Female & $41(41.4)$ & $9(9.3)$ & $50(25.5)$ & $57(47.9)$ & $\begin{array}{c}111 \\
(65.7)\end{array}$ & $168(58.3)$ \\
\hline \multirow[t]{5}{*}{ Age } & Mean & 40.2 & 38.1 & 39.2 & 44.1 & 46.2 & 45.3 \\
\hline & Median & 37 & 35 & 38 & 41 & 44 & 40.5 \\
\hline & Std. Deviation & 10 & 13 & 11.81 & 14.7 & 14.1 & 14.4 \\
\hline & Minimum & 21 & 15 & 15 & 13 & 16 & 13 \\
\hline & Maximum & 68 & 89 & 89 & 95 & 83 & 95 \\
\hline \multirow{4}{*}{$\begin{array}{l}\text { Marital } \\
\text { Status }\end{array}$} & Single & $2(2.0)$ & $4(4.1)$ & $6(3.1)$ & $16(13.4)$ & $18(10.7)$ & $34(11.8)$ \\
\hline & Married & $90(90.9)$ & 89 (91.8) & $\begin{array}{l}179( \\
91.3)\end{array}$ & $92(77.3)$ & $\begin{array}{c}116 \\
(68.6)\end{array}$ & $208(72.2)$ \\
\hline & Widowed & $5(5.1)$ & $3(3.1)$ & $8(4.1)$ & $10(8.4)$ & $31(18.3)$ & $41(14.2)$ \\
\hline & Divorced & $2(2.0)$ & $1(1.0)$ & $3(1.5)$ & $1(0.8)$ & $4(2.4)$ & $5(1.7)$ \\
\hline \multirow[t]{5}{*}{$\begin{array}{l}\text { Education } \\
\text { status }\end{array}$} & $\begin{array}{l}\text { Never attend } \\
\text { school }\end{array}$ & $16(16.2)$ & $12(12.4)$ & $28(14.3)$ & $1(0.8)$ & $9(5.3)$ & $10(3.5)$ \\
\hline & $\begin{array}{l}\text { Informal } \\
\text { school }\end{array}$ & $0(0.0)$ & $1(1.0)$ & $1(0.5)$ & $2(1.7)$ & $0(0.0)$ & $2(0.7)$ \\
\hline & Primary school & $74(74.7)$ & $71(73.2)$ & $145(74.0)$ & $101(84.9)$ & $\begin{array}{c}118 \\
(69.8)\end{array}$ & $219(76.0)$ \\
\hline & $\begin{array}{l}\text { Secondary } \\
\text { school }\end{array}$ & $8(8.1)$ & 13.4 & $21(10.7)$ & $11(9.2)$ & $34(20.1)$ & $45(15.6)$ \\
\hline & $\begin{array}{l}\text { Tertiary } \\
\text { education }\end{array}$ & $1(1.0)$ & $0(0.0)$ & $1(0.5)$ & $4(3.4)$ & $8(4.7)$ & $12(4.2)$ \\
\hline \multirow[t]{6}{*}{$\begin{array}{l}\text { Household } \\
\text { size }\end{array}$} & $1-5$ & $33(33.3)$ & $40(41.2)$ & $73(37.2)$ & $74(62.2)$ & $\begin{array}{c}110 \\
(65.1)\end{array}$ & $184(63.9)$ \\
\hline & $6-10$ & $46(46.5)$ & $52(53.6)$ & $98(50.0)$ & $45(37.8)$ & $59(34.9)$ & $104(36.1)$ \\
\hline & $11-15$ & $14(14.1)$ & $3(3.1)$ & $17(8.7)$ & $0(0.0)$ & $0(0.0)$ & $0(0.0)$ \\
\hline & $16-20$ & $5(5.1)$ & $0(0.0)$ & $5(2.6)$ & $0(0.0)$ & $0(0.0)$ & $0(0.0)$ \\
\hline & $21-25$ & $0(0)$ & $2(2.1)$ & $2(1.0)$ & $0(0.0)$ & $0(0.0)$ & $0(0.0)$ \\
\hline & $>25$ & $1(1.0)$ & $0(0)$ & $1(0.5)$ & $0(0.0)$ & $0(0.0)$ & $0(0.0)$ \\
\hline \multirow{5}{*}{$\begin{array}{l}\text { Reason for } \\
\text { keeping } \\
\text { pigs }\end{array}$} & $\begin{array}{l}\text { Income } \\
\text { generation }\end{array}$ & $99(67.3)$ & $97(93.0)$ & $196(78)$ & $\begin{array}{c}117 \\
(92.13)\end{array}$ & $\begin{array}{c}167 \\
(81.5)\end{array}$ & $284(85.5)$ \\
\hline & Reproduction & $28(19.0)$ & $1(1.0)$ & $29(12)$ & $3(2.36)$ & $0(0.0)$ & $3(0.9)$ \\
\hline & Manure & $2(1.4)$ & $1(1.0)$ & $3(1)$ & $7(5.51)$ & $34(16.6)$ & $41(12.3)$ \\
\hline & $\begin{array}{l}\text { Domestic } \\
\text { consumption }\end{array}$ & $14(9.5)$ & $3(3)$ & $17(7)$ & $0(0.0)$ & $4(2.0)$ & $4(1.2)$ \\
\hline & Cultural & $4(2.7)$ & $2(2)$ & $6(2)$ & $0(0.0)$ & $0(0.0)$ & $0(0.0)$ \\
\hline
\end{tabular}




\section{Socio-Demographic Characteristics of Pig Traders}

A total of 28 pig traders were obtained during data collection. Out of them, seven were from Songwe district, five from Momba district, 11 were from Songea and five were from Mbinga districts (Table 2). All five assembler traders were married males with minimum age 27 and maximum 44 years, and four had acquired primary education. On the other hand, 21 retailer traders were males and two were females with age minimum of 17 years and maximum of 65 years". Over $50 \%$ attended primary education and only two were not married. In order to start pig business, the minimum initial capital investment for selling pork meat was between USD 22 and USD 87 (Table 2).

TABLE 2: Socio-demographic characteristics of pig traders in Songwe and Ruvuma regions, Tanzania

\begin{tabular}{|c|c|c|c|c|c|c|c|c|c|}
\hline \multirow{2}{*}{ Category } & \multirow{2}{*}{ Variable } & \multicolumn{2}{|c|}{ Songwe $(\mathrm{n}=7)$} & \multicolumn{2}{|c|}{ Momba $(n=5)$} & \multirow{2}{*}{$\begin{array}{l}\begin{array}{l}\text { Total } \\
(\mathrm{N}=12)\end{array} \\
\text { No }\end{array}$} & \multirow{2}{*}{$\begin{array}{l}\begin{array}{l}\text { Songea } \\
(\mathrm{n}=11)\end{array} \\
\text { No }\end{array}$} & \multirow{2}{*}{$\begin{array}{l}\text { Mbinga } \\
(n=5) \\
\text { No }\end{array}$} & \multirow{2}{*}{$\begin{array}{l}\text { Total } \\
(\mathrm{N} \\
=16) \\
\text { No }\end{array}$} \\
\hline & & No & No & No & No & & & & \\
\hline & & $\begin{array}{l}\text { Assemblers } \\
(\mathrm{n}=2)\end{array}$ & $\begin{array}{l}\text { Retailers } \\
(n=5\end{array}$ & $\begin{array}{l}\text { Assemblers } \\
(\mathrm{n}=3)\end{array}$ & $\begin{array}{l}\text { Retailers } \\
(n=2)\end{array}$ & & $\begin{array}{l}\text { Retailers } \\
(\mathrm{n}=11)\end{array}$ & $\begin{array}{l}\text { Retailers } \\
(n=5)\end{array}$ & \\
\hline \multirow[t]{5}{*}{ Age } & Mean & 35.5 & 30.6 & 35.7 & 30.5 & 32.7 & 37.5 & 50 & 41.4 \\
\hline & Median & 35.5 & 30 & 35 & 30.5 & 31 & 39 & 48 & 40 \\
\hline & $\begin{array}{l}\text { Std. } \\
\text { Deviation }\end{array}$ & 8.5 & 9 & 4.9 & 1.5 & 7.64 & 7.53 & 10.5 & 10.3 \\
\hline & Minimum & 27 & 17 & 30 & 29 & 17 & 24 & 38 & 24 \\
\hline & Maximum & 44 & 45 & 42 & 32 & 45 & 52 & 65 & 65 \\
\hline \multirow[t]{2}{*}{ Sex } & Male & 2 & 4 & 3 & 2 & 11 & 11 & 4 & 15 \\
\hline & Female & 0 & 1 & 0 & 0 & 1 & 0 & 1 & 1 \\
\hline \multirow[t]{2}{*}{$\begin{array}{l}\text { Marital } \\
\text { status }\end{array}$} & Single & 0 & 1 & 0 & 0 & 1 & 1 & 0 & 1 \\
\hline & Married & 2 & 4 & 3 & 2 & 11 & 10 & 5 & 15 \\
\hline \multirow[t]{3}{*}{$\begin{array}{l}\text { Education } \\
\text { status }\end{array}$} & $\begin{array}{l}\text { Never } \\
\text { attended } \\
\text { school }\end{array}$ & 1 & 1 & 0 & 0 & 2 & 0 & 0 & 0 \\
\hline & $\begin{array}{l}\text { Primary } \\
\text { school }\end{array}$ & 1 & 4 & 3 & 2 & 10 & 10 & 5 & 15 \\
\hline & $\begin{array}{l}\text { Secondary } \\
\text { school }\end{array}$ & 0 & 0 & 0 & 0 & 0 & 1 & 0 & 1 \\
\hline \multirow[t]{2}{*}{$\begin{array}{l}\text { Household } \\
\text { size }\end{array}$} & $1-5$ & 1 & 4 & 2 & 1 & 8 & 7 & 4 & 11 \\
\hline & $6-10$ & 1 & 1 & 1 & 1 & 4 & 4 & 1 & 5 \\
\hline \multirow[t]{4}{*}{$\begin{array}{l}\text { Initial } \\
\text { capital } \\
\text { investment }\end{array}$} & $\begin{array}{l}22-87 \\
\text { USD }\end{array}$ & 0 & 4 & 0 & 2 & 6 & 3 & 0 & 3 \\
\hline & $\begin{array}{l}109-217 \\
\text { USD }\end{array}$ & 0 & l & 0 & 0 & 1 & 8 & 4 & 12 \\
\hline & $\begin{array}{l}>217- \\
435 U S D\end{array}$ & 1 & 0 & 0 & 0 & 1 & 0 & 1 & 1 \\
\hline & $\begin{array}{l}>435 \\
\text { USD }\end{array}$ & 1 & 0 & 3 & 0 & 4 & 0 & 0 & 0 \\
\hline
\end{tabular}




\section{Main Actors in Pig Production Value Chain}

Through the mapping process, it was found that the main actors involved in production, distribution and marketing were pig producers, assemblers, wholesalers, rural and urban retailers. The flow of products involved live pigs, raw pork, offal, heads, legs and pig's oil. Pig producers were involved in rearing pigs and selling directly to traders. Assembler traders were engaged in transportation of live pigs within and outside districts. The wholesale traders were involved in buying and selling pigs in large quantity from assemblers while retail traders were involved in buying live pigs in small quantity from pig producers, slaughtering and selling raw pork or roasted pork to end consumers (Fig 2).

\section{FIGURE 2. Mapping of actors and flow of products in pig value chain in Songwe and Ruvuma regions, Tanzania.}
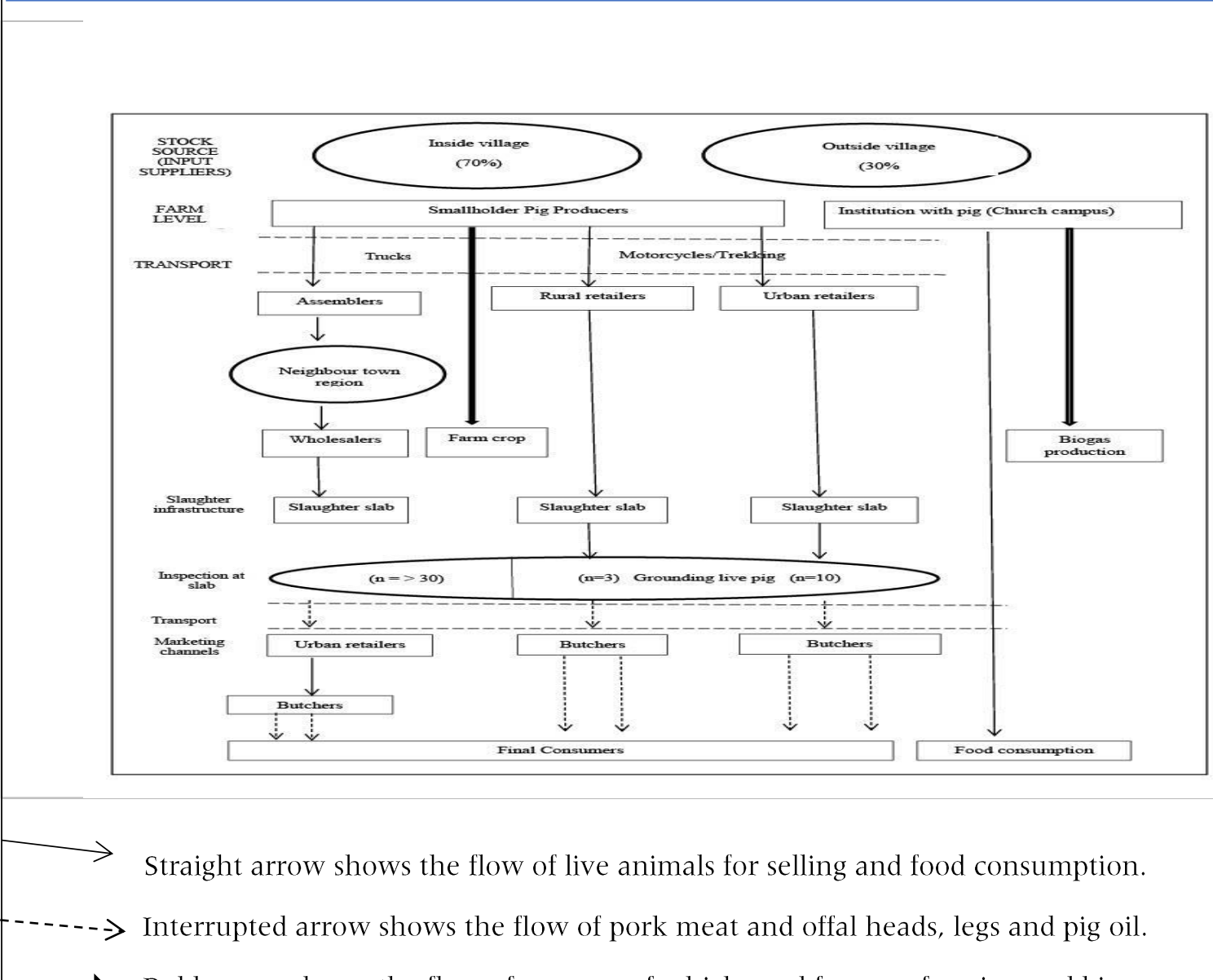

Straight arrow shows the flow of live animals for selling and food consumption.

Interrupted arrow shows the flow of pork meat and offal heads, legs and pig oil.

Bold arrow shows the flow of manure of which used for crop farming and biogas generation.

\section{The Production Node}

\section{Pig Management and Production Practices}

Over $50 \%$ of pig producers reported to have acquired pig stocks from neighboring farms within study areas. However, it was found that producers in Songwe (7; $7.1 \%)$, Momba $(29 ; 29.9 \%)$, Songea $(6 ; 5 \%)$ and
Mbinga (21; 12.4\%) obtained pigs from outside villages and neighboring regions. Majority of pig producers in Songwe (93; 93.9\%), Momba (89; $91.8 \%$ ), Songea (94; 79.0\%) and Mbinga (138; $81.7 \%$ ) preferred to keep indigenous breeds compared to pure breeds, cross breeds and exotic breeds. Most of pig producers in Songwe $(42 ; 42.4 \%)$, Momba (33; 
$34 \%)$, Songea $(81 ; 68.1 \%)$ and Mbinga $(158 ; 94.1 \%)$ owned about 1.00 acres to 2.00 acres for pig production. A few producers practiced free ranging in Songwe $(4 ; 4 \%)$, Momba $(5 ; 5.2 \%)$, Songea $(1 ; 0.8)$; while Semi confinement production system was observed in Songwe $(44 ; 44.4 \%)$ and Momba (66; $68 \%$ ) (Table 3).

TABLE 3. Pig Producers' management and production practices in Songwe and Ruvuma Regions, Tanzania

\begin{tabular}{|c|c|c|c|c|c|c|c|}
\hline \multirow[t]{3}{*}{ Category } & \multirow[t]{3}{*}{ Variables } & \multicolumn{3}{|c|}{ Songwe Region } & \multicolumn{3}{|c|}{ Ruvuma Region } \\
\hline & & \multirow{2}{*}{$\begin{array}{l}\text { Songwe } \\
(\mathrm{n}=99)\end{array}$} & \multirow{2}{*}{$\begin{array}{c}\text { Momba } \\
(\mathrm{n}=97) \\
\end{array}$} & \multirow{2}{*}{$\begin{array}{l}\text { Total } \\
(\mathrm{N}= \\
196) \\
\end{array}$} & \multirow{2}{*}{$\begin{array}{c}\text { Songea } \\
(\mathrm{n}=119)\end{array}$} & \multirow{2}{*}{$\begin{array}{l}\text { Mbinga } \\
(\mathrm{n}=169)\end{array}$} & \multirow{2}{*}{$\begin{array}{c}\text { Total } \\
(\mathrm{N}=288) \\
\end{array}$} \\
\hline & & & & & & & \\
\hline & & No. $(\%)$ & No. (\%) & No. (\%) & No. (\%) & No. (\%) & No. $(\%)$ \\
\hline \multirow[t]{5}{*}{$\begin{array}{l}\text { Source of } \\
\text { stock }\end{array}$} & Neighbouring farm & $85(85.9)$ & $58(59.8)$ & $\begin{array}{c}143 \\
(72.9)\end{array}$ & $96(80.7)$ & $124(73.4)$ & $220(76.4)$ \\
\hline & Outside village & $7(7.1)$ & $29(29.9)$ & $36(18.4)$ & $6(5.0)$ & $21(12.4)$ & $27(9.4)$ \\
\hline & Mission camp & $0(0.0)$ & $0(0.0)$ & $0(0)$ & $14(11.8)$ & $19(11.2)$ & $33(11.5)$ \\
\hline & Family/relative & $6(6.0)$ & $7(7.2)$ & $13(6.6)$ & $1(0.8)$ & $4(2.4)$ & $5(1.7)$ \\
\hline & Others & $1(1.0)$ & $3(2.0)$ & $4(3.0)$ & $2(1.7)$ & $1(0.6)$ & $3(1.1)$ \\
\hline \multirow[t]{3}{*}{ Breed type } & Indegenous breed & $93(93.9)$ & $89(91.8)$ & $182(92.9)$ & $94(79.0)$ & $138(81.7)$ & $232(80.6)$ \\
\hline & Exotic pure breed & $2(2.0)$ & $3(3.1)$ & $5(2.6)$ & $9(7.6)$ & $4(2.4)$ & $13(4.5)$ \\
\hline & Cross breed & $4(4.0)$ & $5(5.2)$ & $9(4.6)$ & $16(13.4)$ & $27(16.0)$ & $43(14.9)$ \\
\hline \multirow[t]{6}{*}{ Herd size } & 0 & $14(14.1)$ & $17(17.5)$ & $31(15.8)$ & $32(26.9)$ & $30(17.8)$ & $62(21.5)$ \\
\hline & $1-4$ & $44(44.4)$ & $31(32.0)$ & $75(38.3)$ & $58(48.7)$ & $106(62.7)$ & $164(56.9)$ \\
\hline & $5-9$ & $20(20.2)$ & $27(27.8)$ & $47(24.0)$ & $17(14.3)$ & $17(10.1)$ & $34(11.8)$ \\
\hline & $10-14$ & $11(11.1)$ & $12(12.4)$ & $23(11.7)$ & $5(4.2)$ & $12(7.1)$ & $17(5.9)$ \\
\hline & $15-19$ & $2(2.0)$ & $3(3.1)$ & $5(2.6)$ & $3(2.5)$ & $1(0.6)$ & $4(1.4)$ \\
\hline & $>20$ & $8(8.1)$ & $7(7.2)$ & $15(7.7)$ & $4(3.4)$ & $3(1.8)$ & $7(2.4)$ \\
\hline \multirow[t]{6}{*}{ Land Size } & Don't own land & $10(10.1)$ & $11(11.3)$ & $21(10.7)$ & $9(7.6)$ & $9(5.3)$ & $18(6.3)$ \\
\hline & $1.00-2.00$ acres & $42(42.4)$ & $33(34.0)$ & $75(38.3)$ & $81(68.1)$ & $159(94.1)$ & $240(83.3)$ \\
\hline & $>2.00-4.00$ acres & $21(21.2)$ & $31(32.0)$ & $52(26.5)$ & $22(18.5)$ & $0(0.0)$ & $22(7.6)$ \\
\hline & $\begin{array}{l}>4.00-10.00 \\
\text { acres }\end{array}$ & $14(14.1)$ & $17(17.5)$ & $31(15.8)$ & $6(5.0)$ & $1(0.6)$ & $6(2.1)$ \\
\hline & $>10$ acres & $12(12.1)$ & $5(5.2)$ & $17(8.7)$ & $1(0.8)$ & $1(0.6)$ & $2(0.7)$ \\
\hline & Confinement & $41(41.4)$ & $26(26.8)$ & $67(34.2)$ & $113(95.0)$ & $169(100)$ & $282(97.9)$ \\
\hline \multirow{3}{*}{$\begin{array}{l}\text { Production } \\
\text { system }\end{array}$} & Semi confinement & $44(44.4)$ & $66(68.0)$ & $\begin{array}{c}110 \\
(56.1)\end{array}$ & $4(3.4)$ & $0(0.0)$ & $4(1.4)$ \\
\hline & Free range & $4(4.0)$ & $5(5.2)$ & $9(4.6)$ & $1(0.8)$ & $0(0.0)$ & $1(0.3)$ \\
\hline & Tethering & $10(10.1)$ & $0(0.0)$ & $10(5.1)$ & $1(0.8)$ & $0(0.0)$ & $1(0.3)$ \\
\hline \multirow[t]{7}{*}{$\begin{array}{l}\text { Who take } \\
\text { care of pigs }\end{array}$} & Husband & $15(15.2)$ & $20(20.6)$ & $35(17.9)$ & $13(10.9)$ & $16(9.5)$ & $29(10.1)$ \\
\hline & Wife & $31(31.3)$ & $27(27.8)$ & $58(29.6)$ & $27(22.7)$ & $87(51.5)$ & $114(39.6)$ \\
\hline & Children & $2(2.0)$ & $3(3.1)$ & $5(2.6)$ & $6(5.0)$ & $9(5.3)$ & $15(5.2)$ \\
\hline & Both $1 \& 2$ & $23(23.2)$ & $24(24.1)$ & $47(24.0)$ & $40(33.6)$ & $26(15.4)$ & $66(22.9)$ \\
\hline & All $1,2 \& 3$ & $27(27.3)$ & $22(22.6)$ & $49(25.0)$ & $22(18.5)$ & $18(10.7)$ & $40(13.9)$ \\
\hline & Hired labour & $1(1.0)$ & $0(0)$ & $1(0.5)$ & $3(2.5)$ & $5(3.0)$ & $8(2.8)$ \\
\hline & Himself/herself & $0(0)$ & $1(1.0)$ & $1(0.5)$ & $4(3.4)$ & $1(0.6)$ & $5(1.7)$ \\
\hline
\end{tabular}




\begin{tabular}{|c|c|c|c|c|c|c|c|}
\hline \multirow[t]{3}{*}{ Category } & \multirow[t]{3}{*}{ Variables } & \multicolumn{3}{|c|}{ Songwe Region } & \multicolumn{3}{|c|}{ Ruvuma Region } \\
\hline & & Songwe & Momba & \multirow{2}{*}{$\begin{array}{l}\text { Total } \\
(\mathrm{N}= \\
196)\end{array}$} & Songea & \multirow{2}{*}{$\begin{array}{l}\text { Mbinga } \\
(n=169)\end{array}$} & Total \\
\hline & & $(\mathrm{n}=99)$ & $(\mathrm{n}=97)$ & & $(\mathrm{n}=119)$ & & $(\mathrm{N}=288)$ \\
\hline & & No. (\%) & No. (\%) & No. (\%) & No. (\%) & No. (\%) & No. (\%) \\
\hline \multirow[t]{2}{*}{$\begin{array}{l}\text { Disposal of } \\
\text { waste } \\
\text { product }\end{array}$} & $\begin{array}{l}\text { Proper (Deep } \\
\text { burial collecting } \\
\text { waste, burning) }\end{array}$ & $44(44.4)$ & $57(58.8)$ & $101(51.5)$ & $32(26.9)$ & $79(46.7)$ & $111(38.5)$ \\
\hline & $\begin{array}{l}\text { Improper } \\
\text { (throwing away, } \\
\text { feed to pigs, } \\
\text { disposal of dead } \\
\text { pigs) }\end{array}$ & $55(55.6)$ & $40(41.2)$ & $95(48.5)$ & $87(73.1)$ & $90(53.3)$ & $177(61.5)$ \\
\hline \multirow[t]{2}{*}{$\begin{array}{l}\text { Boiling feed/ } \\
\text { leftovers }\end{array}$} & Yes & $27(27.3)$ & $26(26.8)$ & $53(27)$ & $42(35.3)$ & $81(47.9)$ & $123(42.7)$ \\
\hline & No & $72(72.7)$ & $71(73.2)$ & $143(73$ & $77(64.7)$ & $88(52.1)$ & $165(57.3)$ \\
\hline \multirow{2}{*}{$\begin{array}{l}\text { Wash/Use of } \\
\text { protective } \\
\text { gears }\end{array}$} & Yes & $25(25.3)$ & $17(17.5)$ & $42(21.4)$ & $71(59.7)$ & $91(53.8)$ & $162(53.3)$ \\
\hline & No & $74(74.7)$ & $80(82.5)$ & $154(78.6)$ & $48(40.3)$ & $78(46.2)$ & $126(43.8)$ \\
\hline \multirow[t]{2}{*}{$\begin{array}{l}\text { Animal } \\
\text { treatment } \\
\text { services }\end{array}$} & Yes & $64(64.6)$ & $37(38.1)$ & $\begin{array}{c}101 \\
(51.5)\end{array}$ & $115(96.6)$ & $146(86.4)$ & $261(90.6)$ \\
\hline & No & $35(35.4)$ & $60(61.9)$ & $95(48.5)$ & $4(3.4)$ & $23(13.6)$ & $27(9.4)$ \\
\hline
\end{tabular}

It was found that wives were source of family labour in Songwe $(31 ; 31.3 \%)$, Momba $(27 ; 27.8 \%)$, Songea $(27 ; 22.7 \%)$ and Mbinga $(87 ; 51.5 \%)$; while very few producers in Songwe $(2 ; 2 \%)$, Momba $(3 ; 3.1 \%)$, Songea rural $(6 ; 5 \%)$ and Mbinga $(9 ; 5.3 \%)$ reported that children were the ones taking care of pigs. Poor implementation of biosecurity measures such as nonuse of protective gears and washing equipment without use of disinfectants were reported in Songwe $(74 ; 74.7 \%)$, Momba $(80 ; 82.5 \%)$, Songea $(48 ; 40.3)$ and Mbinga $(78 ; 46.2 \%)$. Moreover, it was found that, producers in Songwe $(55 ; 55.6 \%)$, Momba (40; $41.2 \%)$, Songea $87(73.1 \%)$ and Mbinga (90; 53.3\%) managed waste products inappropriately by throwing them away. Different proportions of pig producers in Songwe $(35 ; 35.4 \%)$, Momba $(60 ; 61.9 \%)$, Songea ( 4 ; $3.4 \%)$, and Mbinga $(23 ; 13.6 \%)$ had not treated sick animals due to lack of veterinary services (Table 3 ). Different feeds were used to feed pigs in the study areas. Maize ban was highly used in Songwe (95; $38 \%$ ), brew residuals in Momba (41; 17\%), green leaves in Songea $(96 ; 25 \%)$, and hotel leftovers in Mbinga (20; 5\%) (Fig 3). 


\section{FIGURE 3: Type of feeds used to feed pigs in Songwe and Ruvuma regions, Tanzania}

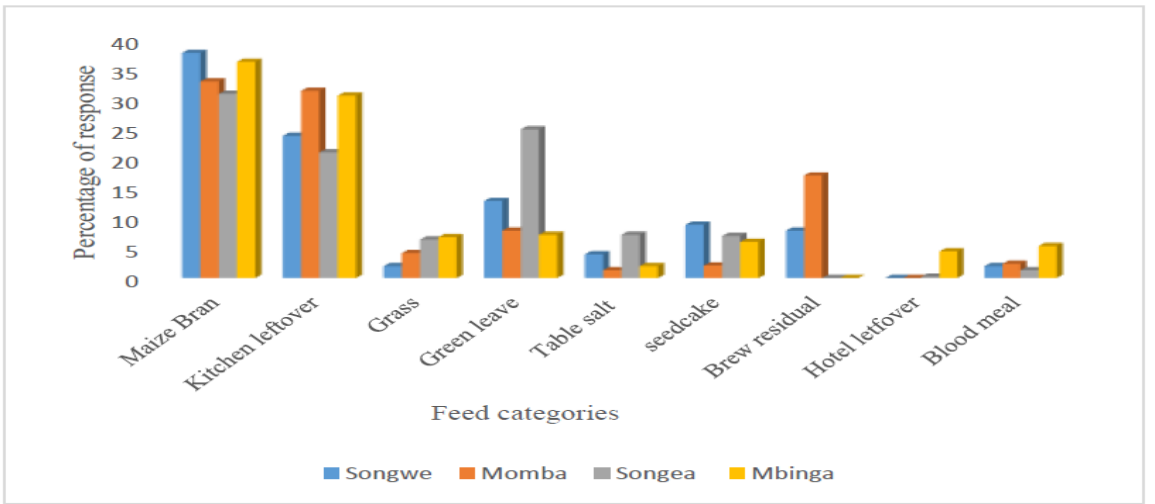

Cooking of pig feeds was not common in Songwe (72; $72.7 \%)$, Momba $(71 ; 73.2 \%)$, Songea $(77 ; 64.7 \%)$ and Mbinga $(88 ; 52.1 \%)$ (Table 3$)$. The common materials used to construct pig premises were grass thatched on roof, timber on walls and soil/timber on floor (Fig. 4a,
$4 \mathrm{~b}, 4 \mathrm{c}, 4 \mathrm{~d})$. Some pig premises (18\%) within the study areas were left open on the roof while $5.0 \%$ to (44; $44.4 \%$ ) had mud floor (Fig 5). The analysis showed that in all districts less than $50 \%$ of pig producers used to clean pig premises on daily or weekly basis (Fig 6).
FIGURE 4a: Peramiho village (Songea District)

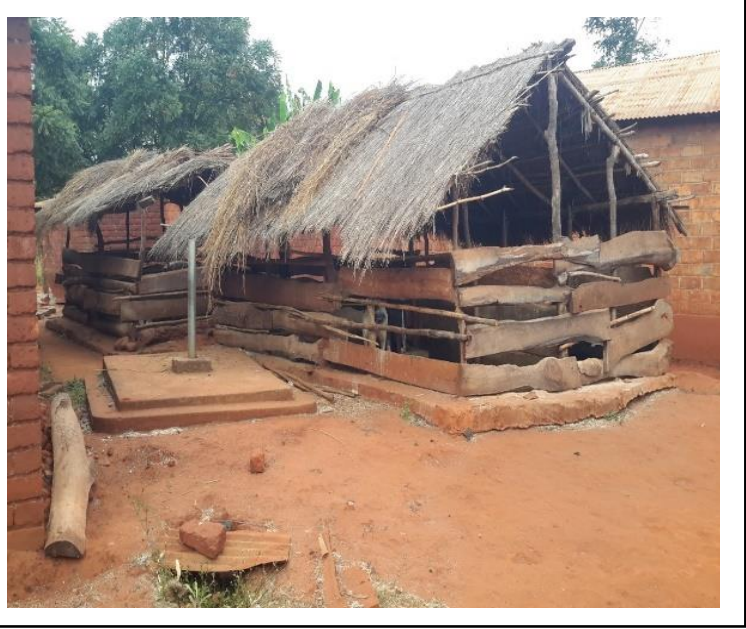

FIGURE 4b: Magagura village (Songea District)

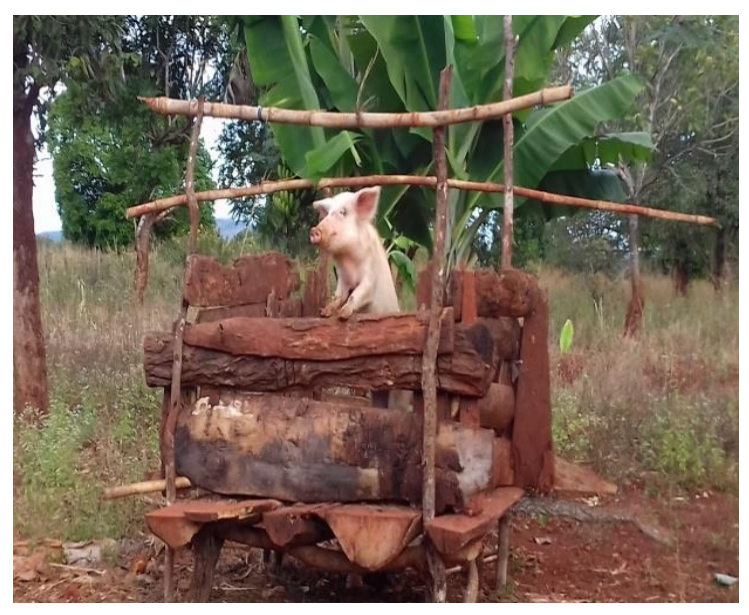



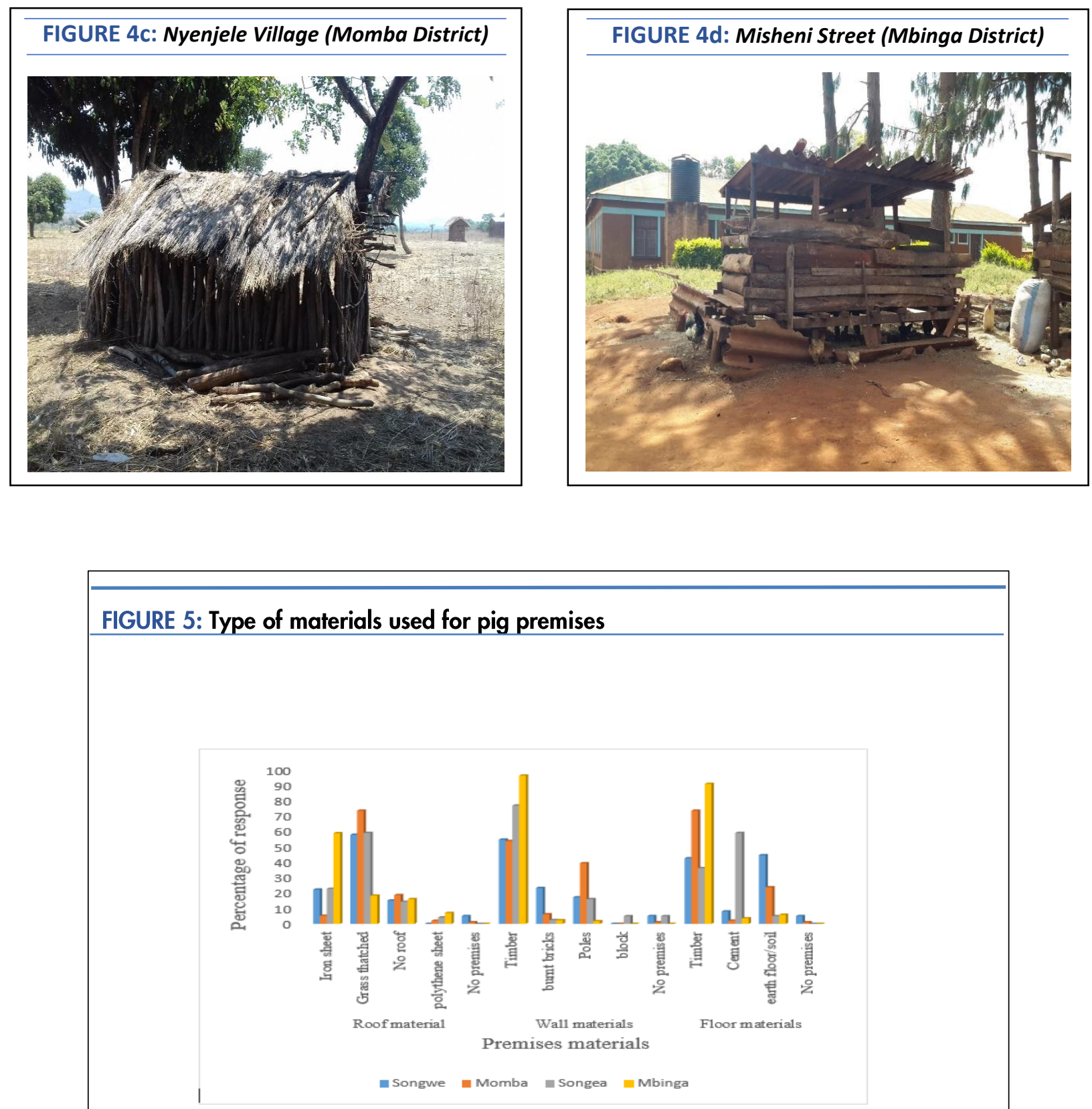


\section{FIGURE 6: Routine cleaning of premises in Songwe and Ruvuma regions, Tanzania.}

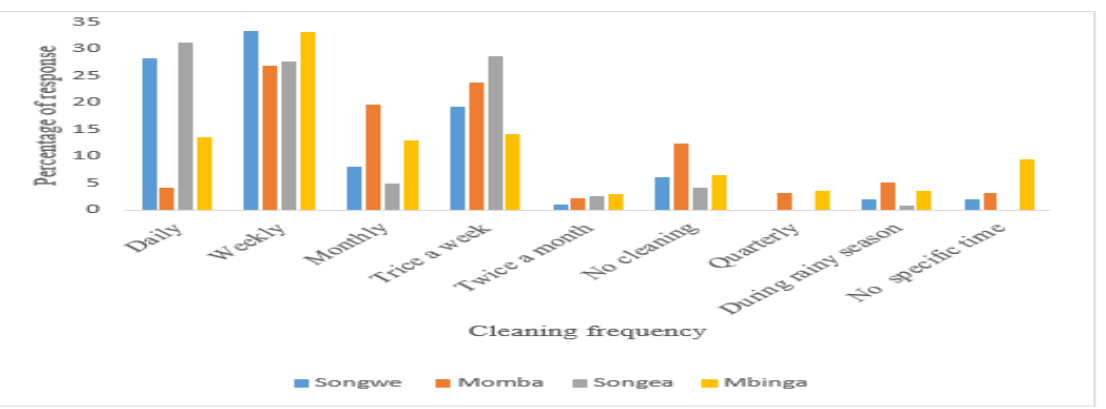

\section{Transportation Node}

\section{Assemblers Marketing Practices}

All 5 surveyed assemblers adopted sole proprietorship form of business, they reported not to have business licenses. Two (2) assemblers lived within study areas and while the rest three (3) came from neighbouring districts and towns in Mbeya and Njombe regions. Assemblers $(n=5)$ purchased live pigs from more than 5 producers and transported them to neighbouring towns/regions. Depending on pig weight, the price ranged between US\$22 and 174. The residential assemblers reported to transport between 6 and 10 live pigs per trip while those from outside the districts transported large numbers of live pigs $(\geq 30)$ per trip. Both residential and non-residential assemblers hired trucks to transport purchased live pigs from study areas to pig slaughter slabs in the neighbouring region/town/city. The assemblers reported to transport live pigs during night and spent an average of 2 hours in short distances of 70 to 100 kilometres and more than 5 hours in long distances of 396.3 kilometres or more. All assemblers reported not to observe bio security measures such as disinfecting the vehicles/trucks before loading and after unloading of animals and during transportation (Table 4).

\section{TABLE 4: Pig traders' marketing activities and practices in Songwe and Ruvuma Regions, Tanzania}

\begin{tabular}{|c|c|c|c|c|c|c|c|c|c|}
\hline \multirow[t]{2}{*}{ Category } & \multirow[t]{2}{*}{ Variable } & \multicolumn{2}{|c|}{ Songwe } & \multicolumn{2}{|c|}{ Momba } & \multirow{2}{*}{$\begin{array}{c}\begin{array}{c}\text { Total } \\
(\mathrm{n}=12)\end{array} \\
\text { No. }(\%)\end{array}$} & \multirow{2}{*}{$\begin{array}{c}\text { Songea } \\
\text { No. (\%) }\end{array}$} & \multirow{2}{*}{$\begin{array}{l}\text { Mbinga } \\
\text { No. (\%) }\end{array}$} & \multirow{2}{*}{$\begin{array}{c}\begin{array}{c}\text { Total } \\
(\mathrm{N}=16)\end{array} \\
\text { No. }(\%)\end{array}$} \\
\hline & & No. $(\%)$ & No. $(\%)$ & No. $(\%)$ & No. $(\%)$ & & & & \\
\hline & & $\begin{array}{l}\text { Assembler } \\
\quad(\mathrm{n}=2)\end{array}$ & $\begin{array}{l}\text { Retailers } \\
\qquad(\mathrm{n}=5\end{array}$ & $\begin{array}{c}\text { Assembler } \\
\quad(n=3)\end{array}$ & $\begin{array}{l}\text { Retailers } \\
\qquad(\mathrm{n}=2)\end{array}$ & & $\begin{array}{l}\text { Retailers } \\
(\mathrm{n}=11)\end{array}$ & $\begin{array}{c}\text { Retailers } \\
(n=5)\end{array}$ & \\
\hline \multirow[t]{2}{*}{$\begin{array}{l}\text { Business } \\
\text { Ownership }\end{array}$} & $\begin{array}{l}\text { Sole } \\
\text { proprietorship }\end{array}$ & $2(100)$ & $4(80.0)$ & $3(100)$ & $2(100)$ & $11(91.7)$ & $11(68.7)$ & $5(31.3)$ & $16(100)$ \\
\hline & Partnership & - & $1(20.0)$ & - & - & $1(8.3)$ & - & - & - \\
\hline \multirow[t]{2}{*}{ License } & Yes & - & - & - & - & - & $7(63.6)$ & $5(100)$ & $12(75.0)$ \\
\hline & No & $2(100)$ & $5(100)$ & $3(100)$ & $2(100)$ & $12(100)$ & $4(36.4)$ & - & $4(25.0)$ \\
\hline \multirow[t]{2}{*}{$\begin{array}{l}\text { Residential } \\
\text { Status }\end{array}$} & $\begin{array}{l}\text { Within } \\
\text { district/urban }\end{array}$ & $1(100)$ & $5(100)$ & - & $2(100)$ & $8(66.7)$ & $11(100)$ & $5(100)$ & $16(100)$ \\
\hline & $\begin{array}{l}\text { Outside } \\
\text { district/urban }\end{array}$ & $1(100)$ & - & $3(100)$ & - & $4(33.3)$ & - & - & - \\
\hline
\end{tabular}




\begin{tabular}{|c|c|c|c|c|c|c|c|c|c|}
\hline $\begin{array}{l}\text { Source of } \\
\text { stock }\end{array}$ & $\begin{array}{l}\text { Both within } \\
\text { and outside }\end{array}$ & $2(100)$ & $5(100)$ & $3(100)$ & $2(100)$ & & $11(100)$ & $5(100)$ & $16(100)$ \\
\hline $\begin{array}{l}\text { Number of } \\
\text { producers }\end{array}$ & $>5$ & $2(100)$ & $5(100)$ & $3(100)$ & $2(100)$ & $12(100)$ & $11(100)$ & $5(100)$ & $16(100)$ \\
\hline \multirow{5}{*}{$\begin{array}{l}\text { Number of } \\
\text { live pigs } \\
\text { purchase }\end{array}$} & 1 & $0(0)$ & $3(60.0)$ & $0(0)$ & $2(100)$ & $5(41.7)$ & $7(63.6)$ & $1(20.0)$ & $8(50.0)$ \\
\hline & $>1-5$ & $0(0)$ & $2(40.0)$ & - & - & $2(16.6)$ & $4(36.3)$ & $4(80.0)$ & $8(50.0)$ \\
\hline & $6-10$ & $2(100)$ & - & - & - & $2(16.7)$ & - & - & - \\
\hline & $>10-30$ & - & - & - & - & - & - & - & - \\
\hline & $>30$ & - & - & $3(100)$ & - & $3(25.0)$ & - & - & - \\
\hline \multirow[t]{3}{*}{$\begin{array}{l}\text { Means of } \\
\text { Transport }\end{array}$} & Trekking & - & $2(40.0)$ & - & $1(50.0)$ & $3(25.0)$ & - & - & - \\
\hline & Hired truck & $2(100)$ & - & $3(100)$ & - & $5(41.7)$ & - & - & - \\
\hline & Motorcycle & - & $3(60.0)$ & - & $1(50.0)$ & $3(33.3)$ & $11(100)$ & $(5(100)$ & $16(100)$ \\
\hline \multirow{3}{*}{$\begin{array}{l}\text { Number of } \\
\text { hours on } \\
\text { road }\end{array}$} & $30-45$ mins & - & $5(100)$ & - & $2(100)$ & $7(58.3)$ & $7(63.6)$ & $3(60.0)$ & $10(62.5)$ \\
\hline & $1-2$ hours & $2(100.0)$ & - & - & - & $2(16.7)$ & $4(36.4))$ & $2(40.0)$ & $6(37.5)$ \\
\hline & $>5$ hours & - & - & $3(100)$ & - & $3(25.0)$ & - & - & \\
\hline \multirow[t]{3}{*}{$\begin{array}{l}\text { Frequency } \\
\text { of purchase }\end{array}$} & $\begin{array}{l}4-10 \\
\text { days/month }\end{array}$ & $2(100)$ & - & $3(100)$ & - & $5(41.7)$ & - & $1(20.0)$ & $1(6.3)$ \\
\hline & $\begin{array}{l}\text { 30days/month } \\
\mathrm{s}\end{array}$ & - & $3(60.0)$ & - & - & $3(25.0)$ & $6(54.5)$ & $4(80.0)$ & $10(62.5)$ \\
\hline & Once/week & - & $2(40.0)$ & - & $2(100)$ & $4(33.3)$ & $5(45.5)$ & - & $5(31.2)$ \\
\hline \multirow[t]{2}{*}{$\begin{array}{l}\text { Protective } \\
\text { gears }\end{array}$} & Yes & - & - & - & - & $1(8.3)$ & $3(27.3)$ & $1(20.1)$ & $4(25.0)$ \\
\hline & No & $2(100)$ & $5(100.0)$ & $3(100)$ & $2(100)$ & $11(91.7)$ & $8(72.7)$ & $4(80.0)$ & $12(75.0)$ \\
\hline
\end{tabular}

\section{Marketing Node}

\section{Wholesalers and Retailers Marketing Practices}

The distribution and marketing of live pigs and pig products involving wholesaler traders were not common in all study districts. However, it was mentioned by assemblers that wholesalers could be found at pig slaughter slabs in neighbouring town/city/region, that is to say, in Mbeya and Njombe regions. In all districts, the retailer traders shared common practices. Majority of pig retailers $(\mathrm{n}=22)$ operated in sole proprietorship while a few operated in partnership. Retailer traders $(n=7)$ had no business licenses, while 12 had business licenses. Traders reported to purchase live pigs from pig producers both within and outside their villages of residence. Retailers $(\mathrm{n}=13)$, purchased live pigs daily, slaughtered and sold raw pork or roasted pork. Depending on the distance, several means of transport including trekking/ motorcycle/bicycle and trucks were used to transport live pigs from farm to different destinations within the study districts. In all districts, retailers were purchasing at least one live pig. Besides, 2 retailers in Songwe, 4 in Songea and 4 in Mbinga reported to purchase more than one live pigs and kept them at home before slaughtering. Slaughtering practices were conducted at slaughter slabs. It was observed that the slabs were open structures, without water supply and other relevant equipment and had poor waste products management. It was observed that retailer traders $(n=4)$ were in gumboots and white overalls at slaughter slabs. The infrastructures and equipment used in butcheries and pork roast shops were of poor hygiene (unclean environment) as they could not protect pork meat from external contamination (Table 4).

\section{Challenges in Prevention and Control of ASF}

The respondents (Livestock Field Officers) reported on poor husbandry practices such as free ranging as well as low bio security measures at farm level and marketing channels. In order to improve production 
and preventing disease outbreaks, application of livestock policy and bylaws within the chain is a necessity. The respondents mentioned presence of animal movement policy, bylaws against free ranging practices, inspection checkpoints, and penalty for violation of by laws. However, it was claimed that monitoring and implementation of the bylaws and regulations was the major challenge. One respondent had this to say: 'During ASF outbreak in 2018, we caught a retailer trader selling pork that was not inspected by livestock in charge officer. We reported the case to the Police Station. However, the suspect won the case due to lack of strong evidence against him' (FLO, Songea district).

Moreover, the respondents reported that there were backyard-slaughtering practices especially during ASF outbreaks. For instance, one respondent reported that I caught a trader who slaughtered at backyard and sold out pork unsuitable for human consumption but due to bureaucracy with long chain of procedures, that trader was able to run away' (FLO, Mbinga district).

In addition, it was reported that the sources of disease outbreaks included farm visitors, animal movements and products from infected villages. One of the respondent had this to say: In order to ensure implementation of animal movement regulations; the Ministry of Livestock and Fisheries should consider to delegate issuing of animal permit to Village Government Authority in order to control illegal movement of animals' (Extension officer, Songwe district).

\section{FINDINGS}

The findings from this study have shown that there are many actors in pig industry from production, distribution, marketing to consumers. Basing on pig value chain performance within districts, the production was dominated by pig producers keeping 1 to 4 pigs. This finding is consistent with census report on pig production in Tanzania. ${ }^{18}$ Many pig producers owned small pieces of land ranging from 1 to 2 acres, this is considered to be enough space to keep 1 to 4 pigs for small scale production. An interesting finding was the fact that only one type of breed was kept by pig producers in all the 4 districts. Many preferred to keep indigenous breeds with a reason that they are less susceptible to diseases including ASF.

The production chain involving pig producers highlighted key information that could contribute to transmission of ASF. Outsourcing pig stocks from various places without considering the health status of pigs could have been the source of ASF introduction and spread in the 4 study districts. ASF transmission through unknown sources has also been reported in previous studies in Uganda ${ }^{23,24}$ and Nigeria. ${ }^{25}$ It was found that practices such as free ranging system during dry season were common in rural areas compared to urban areas. Probably this observation could partly explain the spread of previous outbreaks by free roaming pigs in rural areas. This practice is similar with the characteristic of smallholder producers in Uganda. ${ }^{26}$

Other practices such as low bio security level in pig premises could contribute to introduction of ASF into un-infected pig herds. Pig premises were observed to be very dirty even those in which pig producers claimed to perform daily cleanliness, waste products were maintained nearby the farm. The open roof structures and those with mud floor have a high chance of exposing pigs to a number of infections. These findings are in agreement with those of Dione ${ }^{26}$ in Uganda who reported that open pig premises exposed animals to predators and stray animals. Different feed types were used to feed pigs but yet there was poor handling of the feeds, use of dirty equipment, and feeding pigs on leftovers could also be a source of farm outbreaks. It seems that majority of pig producers have inadequate knowledge on implementation of bio security measures suggesting that purposive education and awareness campaigns should be conducted. Similar findings were reported in Tororo and Busia districts in Uganda and Busia and Teso district in Kenya. ${ }^{24}$

In our study districts, pig marketing was dominated by male traders, implying that there is high disease risk in marketing channels since males are less likely to implement bio security measures. This finding is supported by a study in Uganda that reported a belief by males that bio security measures such as cleanliness are women responsibilities. ${ }^{27}$

Small capital investment to purchase live pigs was observed to be the main concern of pig traders in marketing activities. Along with this, lack of license among pig traders was common in all the study districts. The absence of business licensing especially in rural areas result in lack of data on pig marketing channels, making it nearly impossible for industry planning and development as well as in intervention against ASF.

Transportation of live pigs during night hours could be the source of disease transmission from infected areas to uninfected areas. This finding is consistent with what was found in a previous study in Uganda which reported on behaviour of traders to avoid inspection as 
a loophole for transporting infected pigs. ${ }^{26}$ Moreover, the practice of collecting live animals from more than one farms/producers, loading and unloading of animals without cleaning and disinfection of vehicles could be sources of disease transmission within and outside the districts. Similar observations have been reported in Kenya and Uganda. ${ }^{28}$ Meanwhile, long transportation periods carried high disease risks of infected pigs to affect health pigs as previously reported in Uganda. ${ }^{29}$

Marketing activities of pork meat and other products were more common in urban areas compared to rural areas meaning that marketing activities are economically driven by urbanisation and income growth. On average, the urban retailers slaughtered 10 pigs daily compared to 3 pigs in rural areas. This is an indication that traders in urban areas practice more farm visits due to consumers demand. Additionally, this observation entail that the emerging towns and cities require strict measures to fight against ASF. This is because most towns and cities are targeted market for pork meat.

Poor hygiene was observed in slaughter slabs. Waste products were discarded in open areas and this could be the source of disease outbreaks. Apart from facilitating disease spread, unhygienic environment in slaughter slab, as well as roast shops and butchers, indicate that there is a high chance of consumers to end up with unsafe pork meat.

husbandry system, sharing boars, feeding pork leftovers, and poor disposal of dead pigs to all actors involved in pig production and marketing. The formation of pig business associations should be encouraged to provide an opportunity to improve pig value chain operations and ensure access to market information. Moreover, improved availability and accessibility to veterinary services are likely to play major roles in reducing disease outbreaks through

i) It was conducted when there were no ASF outbreak cases. Although its findings could inform chains operation and sources of ASF outbreaks, more attention should be given to pig chain performance during ASF outbreaks,

ii) The time interval is unlikely to have had influence on practices and there was no interventions implemented in the study areas during the period. However, we cannot account on unknown sources of changes in the practices and their impacts such as exchanges of information among the actors/pig owners between the study sites,

iii) Poor record keeping practices by pig producers and pig traders. Despite being able to capture production practices within the pig value chain; it was not possible
The key informants highlighted that behaviour of traders to avoid animal inspection and slaughtering practice in the backyard played great role in the spread of ASF. This finding entailed the need to improve good animal husbandry practices in order to enhance productivity and meat safety. Meanwhile, field officers pointed out that among the factors that hinder control of animal movements could be the limited involvement of livestock officers and extension officers regarding the issuance of animal movement permits. This weakened enforcement of animal movement control has been attributed to spread of diseases in many countries of Africa and in the Middle East. ${ }^{30}$ Recommendation on decentralised power in issuing the animal movement permits was given as a way forward in solving animal control movements. Parallel to this, quarantine of animals that are being moved between regions and animals introduced within regions should be emphasised in order to prevent the introduction and spread of diseases.

\section{Recommendations to Future Interventions}

Since there is no effective treatment available against ASF, prevention would be much achieved by enforcement of strict bio security measures. It is important to increase awareness on risk practices such as free-ranging

routine disease surveillance, training and extension services. Training should provide knowledge transfer on good husbandry such as confinement of pigs to avoid free-ranging practices associated with outbreaks. Lastly, it is important to improve infrastructures such as slaughter facilities not only to control diseases but also to ensure meat safety.

\section{Study Limitations}

to establish relationship between pig management and factors influencing pig productivity due to absence of records on different variables including the number of piglets born, number of births and age of sow.

\section{CONCLUSIONS}

The findings demonstrated that pig producers, assemblers, and retailer traders were operating in pig production, distribution and marketing chains in Tanzania. Each node performed under poor bio security measures, poor infrastructure and facilities which could contribute to introduction and spread of ASF. The transportation node carried a high disease risk due to many movements and pick-ups. There is need to improve good husbandry practices and marketing 
infrastructures to increase productivity and ensure pork safety. It is anticipated that an overview information on chains operation and possible disease risk practices from this study will be used as inputs in the design of future ASF prevention and control measures in Tanzania.

Acknowledgement: Authors wish to thank Dr. Leonard Mboera for his invaluable inputs in the manuscript as well as indebted to all respondents and actors of pig value chains in Songwe, Momba, Songea and Mbinga districts. This study was funded by the Tanzanian Government and the World Bank through the SACIDS Foundation for One Health with grant number PAD 1436.

\section{REFERENCE}

1. FAO. Meat Market Review.; 2018. http://www.fao.org/3/I9286EN/i9286en.pdf. Accessed 25 July 2019.

2. FAO. Food Outlook: 2018.

3. FAO. Preparation of African Swine Fever Contingency Plans. Vol FAO Animal.; 2009.

4. Maziku M, Desta S, Stapleton J. Pork Production in the Tanzanian Livestock Master Plan. Brief No. 6. ILRI.2017; 4pp

5. Kimbi, Eliakunda C.; Mlangwa, James; Thamsborg, Stig Milan; Mejer, Helena; Lekule F, P. Smallholder Pig Marketing Systems in the Southern Highlands of Tanzania. J Nat Sci Res. 2016; 6(14):8798.

6. FAO/ILRI/AU. Regional Strategy for the Control of African Swine Fever in Africa.; 2017

7. Dixon, L. K., Escribano, J. M. Martins, C. Rock, D. L., Salas, M. L. Wilkinson, P. J. Virus taxonomy. In: Report of the International Committee on Taxonomy of Viruses. (Edited by Fauquet, C. M., Mayo, M. A., Maniloff, J., Desselberger, U. and Ball, L. A.), Elsevier Academic Press, London. 2005. pp. 135 - 143.

8. Health WO for A. African Swine Fever General Disease Information Sheets What Is African Swine Fever? General Disease Information Sheets Where Is the Disease Found?: 2011.

9. Montgomery, R. E. On a form of swine fever occurring in British East Africa (Kenya Colony). J. Comp Pathol 1921. 34: 159 - 191.

10. Penrith ML, Vosloo W. Review of African swine fever: transmission, spread and control. J.S Afric Vet Assoc. 2009; 80(2):58-62.

11. Taylor N, Rushton J. A value chain approach to animal diseases risk management: technical foundations and practical framework for field application. FAO; 2011.

12. Kaplinsky R, Morris M. A HANDBOOK FOR VALUE CHAIN An Important Health Warning or A Guide for Using This Handbook.; 2001. doi:10.1057/9781137373755.0007

13. Porter, M. E. Competitive Advantage, Creating and Sustaining Superior Performance. The Free Press, New York. 32pp; 1985.

14. Gereffi G, Korzeniewicz M, editors. Commodity chains and global capitalism. ABC-CLIO; 1994.

15. Misinzo, G., Kwavi, D. E., Sikombe, C. D., Makange, M., Peter, E., Muhairwa, A. P. and Madege, M. J. Molecular characterization of African swine fever virus from domestic pigs in northern Tanzania during an outbreak in 2013. Trop Anim Health Prod. 2014 46(7): 1199-1207.
16. Misinzo, G., Kasanga, C. J., Mpelumbe-Ngeleja, C., Masambu, J., Kitambi, A. and Van Doorsselaere, J. African swine fever virus, Tanzania, 2010-2012. Emerg Infect Dis. 2012;18(12): 20 - 81 .

17. Wambura, P. N., Masambu, J. and Msami, H. Molecular diagnosis and epidemiology of African swine fever outbreaks in Tanzania. Vet. Res Comm. 2006;30(6): 667-672.

18. United Republic of Tanzania (URT). National Sample Census of Agriculture Small Holder Agriculture. NBS. 2012; 187pp.

19. United Republic of Tanzania (URT). Songwe Region SocioEconomic Profile. NBS. 2015; 205pp.

20. Kothari, C. R. Research Methodology; Methods and Techniques. (2nd Ed); 2004.

21. Thrusfield M. Veterinary epidemiology. John Wiley \& Sons; 2018 Apr 30.

22. Wilson TR. The White Meat Value Chain in Tanzania-a Report from the Southern Highlands Food Systems Programme. FAO; 2015. pp 138.

23. Kabuuka T, Kasaija PD, Mulindwa H, Shittu A, Bastos ADS, Fasina FO. Drivers and risk factors for circulating African swine fever virus in Uganda, 2012-2013. Res Vet Sci. 2014;97(2):218-225. doi:10.1016/j.rvsc.2014.07.001

24. Nantima N, Ocaido M, Ouma E, et al. Risk factors associated with occurrence of African swine fever outbreaks in smallholder pig farms in four districts along the Uganda-Kenya border. Trop Anim Health Prod. 2015;47(3):589-595. doi:10.1007/s11250-015-07689

25. Fasina FO, Agbaje $M$, Ajani FL et al. Risk factors for farm-level African swine fever infection in major pig-producing areas in Nigeria, 1997-2011. Prev Vet Med. 2012;107(1-2):65-75. doi:10.1016/j.prevetmed.2012.05.011

26. Dione MM, Akol J, Roesel K, et al. Risk Factors for African Swine Fever in Smallholder Pig Production Systems in Uganda. Transbound Emerg Dis. 2017;64(3):872-882. doi: $10.1111 /$ tbed. 12452

27. Dione MM, Ochago R, Ouma EA, Lule PM, Kakinda MJ, Nyapendi R, Birungi R, Pyburn R. Gendered perceptions of biosecurity and the gender division of labor in pig farming in Uganda. Journal of Gender, Agriculture and Food Security. 2020

28. Jacqueline A, Lichoti K, Davies J, et al. Pig traders' networks on the Kenya-Uganda border highlight potential for mitigation of African swine fever virus transmission and improved ASF disease risk management. Prev Vet Med. 2017. doi:10.1016/j.prevetmed.2017.03.005

29. Dione M, Ouma E, Opio F, Kawuma B, Pezo D. Qualitative analysis of the risks and practices associated with the spread of African swine fever within the smallholder pig value chains in Uganda. $\begin{array}{lll}\text { Prev Vet } & \text { Med. }\end{array}$ doi:10.1016/j.prevetmed.2016.11.001

30. Ogundipe GAT (2002). The roles of veterinary quarantine services in monitoring the movements.pdf. Niger Vet J. 2002;23 (1):1-15

\section{Peer Reviewed}

Competing Interests: None declared

Received: 2/18/2020; Accepted: 3/22/2021

Cite this article as: Ngosomwile N, Sindato C, Komba VGE, Ndyetabula WD. The role of Pig production and Market value chain in the occurrence of African Swine Fever in Songwe and Ruvuma regions, Tanzania. East Afr Science J. 2021: 3(1): 86-101. https://doi.org/10.24248/EASci-D-20-00004

(C) Ngosomwile et al. This is an open-access article distributed under the terms of the Creative Commons Attribution License, which permits unrestricted use, distribution, and reproduction in any medium, provided the original author and source are properly cited. To view a copy of the license, visit http://creativecommons.org/licenses/by/4.0/. When linking to this article, please use the following permanent link: https://doi.org/10.24248/EASci-D-20-00004 